\title{
$\Im \mathfrak{n} \mathfrak{h a l t}$.
}

\section{B̈̈rgerltues Medit.}

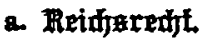

Rx.

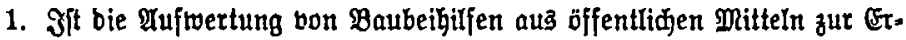
bauung von Bergmannswohnhäufern burd \$29 ber Iritten Steuer.

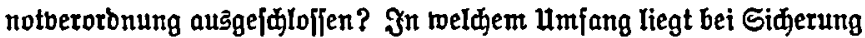

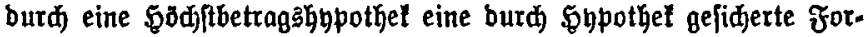

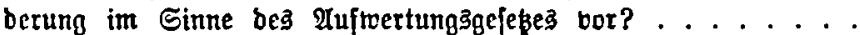

2. 2ujređnung bei gegenfeitigen Forberungen aus borfäglid) begangener unetlaubter \$anblung . . . . . . . . . . . . . .

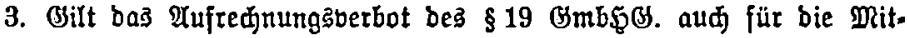

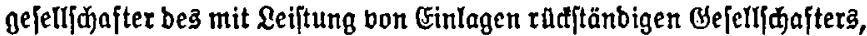
bie zur 8ahlung herangezogen werben jollen? .........

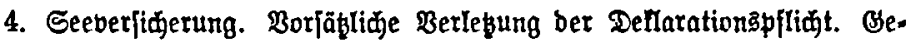
fahrerbeblide umptānde ...............

5. Seeberficherung. Berfiderungarifito bei laufenber Berfiøerung. Allgemein belannte umitänbe im Sinne bon $\$ 19$ ber arlg. beutjđen Secberficerungabebingungen .

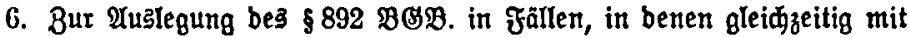

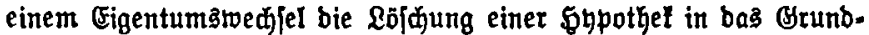

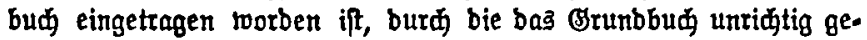
worben fein foll ...................

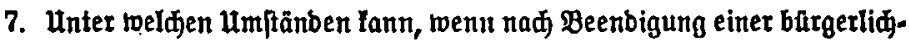

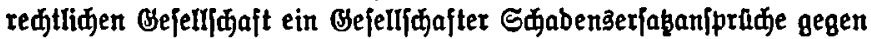
einen anbern erhebt, bon ber vorkerigen Finleitung bes Ausetnamberfeß̧unģberfahrens abgejehen werben? .......... 
9ir.

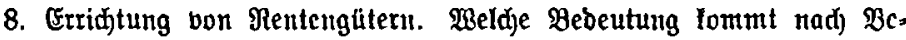

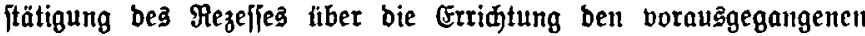
Berbandlungen zu? Sann noh bie Nidtigfeit eines bem Rezeß zugrumbe gelegten notariellen STaufbertraga wegen unrid)tiger $\mathfrak{B} e$ urfunbung beg Saufpreifes geltend gemadht merben? . . . . . . .

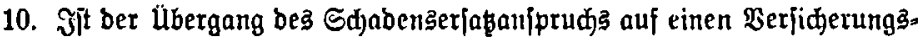

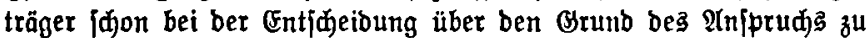

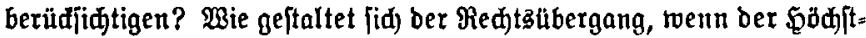

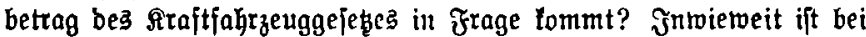
ber f̧eftfebung einer ङめabenşrente lünftigen ßeränberunge॥ Ređjnung zu tragen?...................

11. Entfđäbigung für Enteignung. Bebeutung eines in einem Borprozeß ergangenen red)täfräjtigen Urteils über eine unriđtig bered)utc

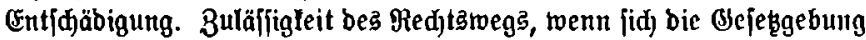
barüber geänbert hat

12. Stann eine eingetragene $\mathfrak{Q}($ uflaffungabornterfung twegen bes öffentlid)en Blaubens besె (Yrunbbud)క nuđ) zum Radteile beffen geltend gemad)t werben, zu befien Bunjten fie berwilligt wurbe? $\mathfrak{M a n u}$ ift bie $\mathfrak{A b}$ löjung eimer $\mathfrak{s y p o t h e l ~ a l s ~ f u ̈ r ~ \Re e d n u n g ~ b e s ~ ( E r w e r b e r a ~ e r f o l g t ~ a l l = ~}$ zufehen? . . . . . . . . . . . . . . . .

13. übernahme bes ßermögens nad) $\$ 419 \mathscr{B}(393$; übertragung ber bazu gchjörigen Gregenjtände einzeln an mehrere łerjonen. Difentlidjer ßlaube besె (अrunbbud)s beim (Erwerb burd) eine Bermögcns̈übernahmc, namentlidi bei bormeggenommener Erbjhaft . . . . . . . . 52

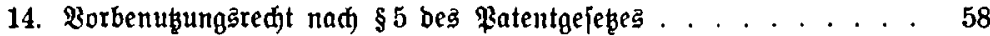

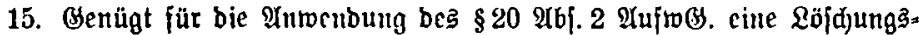
bewilligung audj bann, wenn fie wegen bes binglidjen Ried)ts eiren Borbefalt entjält? . . . . . . . . . . . . . .

16. Saum ein \$ypothefengläubiger nod) Alupertung fraft Sorbehalts ver langen, wenn burd redtsträftiges urteil fejtgeftellt ift, bnßi jeine Forberung burळ Bahlung ifres Mennbetrags in Papiermart getilgt fei?

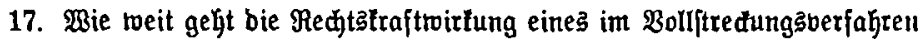

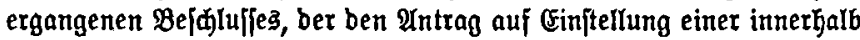

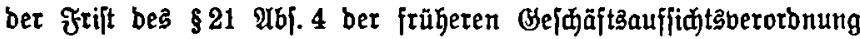
vorgenommenen 8roangsodlltredung zurüđgetwiejen hat? Sönnen

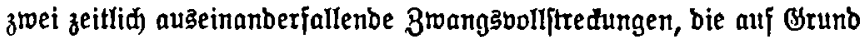


$\mathfrak{n x}$

Gette

besjelben Bollftredungstitela erfolgt find, für bie Bered)unng ber be= zeidjneten Frift als eine einheitlidje 3 roangşvollftredungsmmafnahme betradjtet werben? . . . . . . . . . . . . . . .

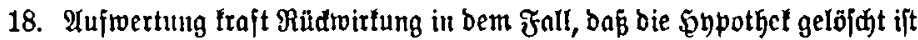
unb igrer $\mathfrak{B i e b e r e i n t r a g u n g ~ b e r ~} \mathfrak{H m}$ itano entgegenjteht, baß ein Cigens tumabrud)teil bes Brumbjtüds inzwijđen alt einen gutgläubigen $\mathrm{Fr}_{\text {r }}$ werber veräuß̧ert ijt .

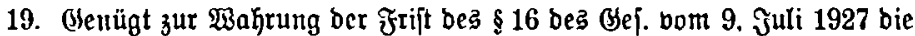

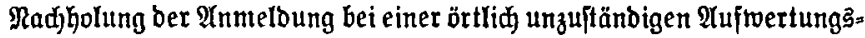
ftelle? . . . . . . . . . . . . . . .

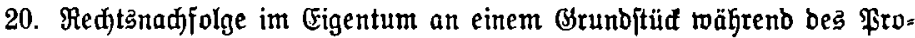

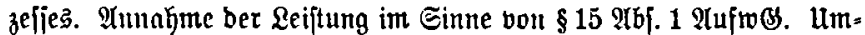

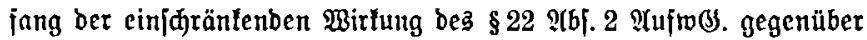

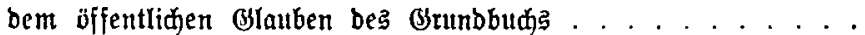

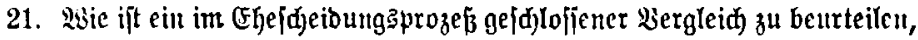

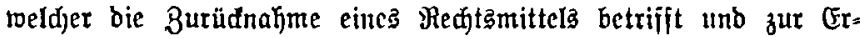
leid)terung bor Sdjeibung bienen foll?

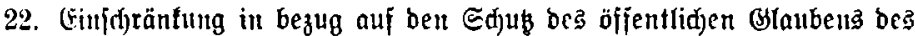

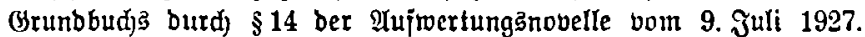

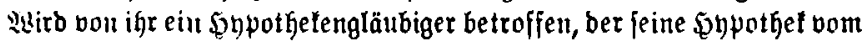
(Grumbtüđäerwerber bor bem Jnfrajttreten ber Rovelle erworben hat?

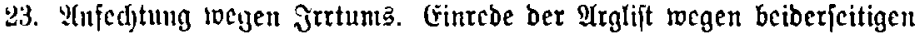
Jrrtums gegeniiber cinten Berlangen nad Afufwertung fraft Rüd wirfuttg

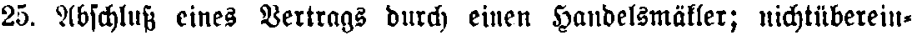
ftimmenbe Mitteilungen bon ifm an beibe Bertragäteile. Jnnmietweit twerben Borbehalte fiber Entbinbung bon ber Reiftungapflid)t bei

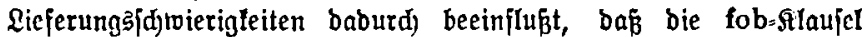
beigefügt wirb . . . . . . . . . . . .

26. Stann ber Gejelljdjafter einer Bejelljdjaft mb. nad ifrer Gintragung

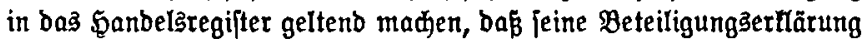
wegen fittentwibrigen Bethaltena einea Mitgejelljdafters bei ber Brünbung unwirffam fei? . . . . . . . . . . . . . . . 102

27. Über IItt und umfang ber Bertretungsbefugnis bes Sorrefpondent=

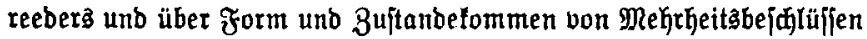
ber Reeberei ..................... 
grt.

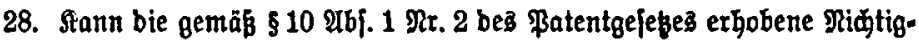
feitallage auf ein bernidtetes \$atent geftübt werben? . . . . 113

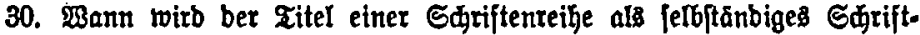
werl gejúbt? ..................

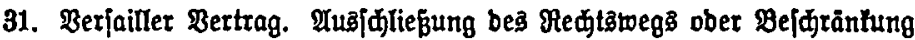
ber Buftänbigfeit beutjüer Beridte? Uufwertung einer forberung, für bie eine $\mathfrak{g}$ ypothel auf einem an \$olen gejallenen Brunbftü beftellt twar? ....................130

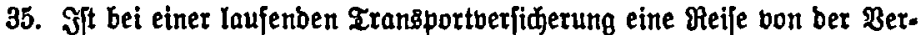
fidjerung gebeat, wenn nur ber Beginn ber Reife in bie Berjidjerungä.

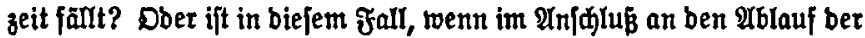
biakherigen Berjiderung eine neue Iaufenbe Berfidjerung genommen wurbe, bie Reife burd biefe gebedt? . . . . . . . . .

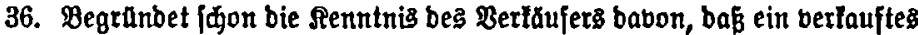

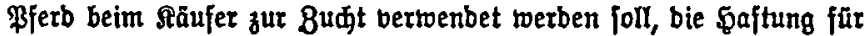

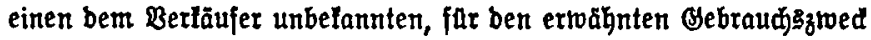
erkeblidjen frehler? . . . . . . . . . . . . . 147

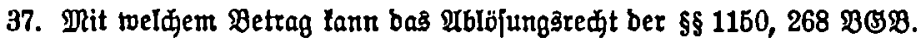
gegenuber einer aufgetwerteten Џodfftbettag

38. Bie wirtt bei einer offenen \$anbels̈gejelljajaft, bie zum Bsegenftanb

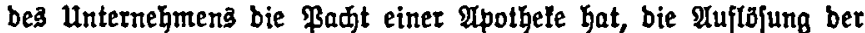

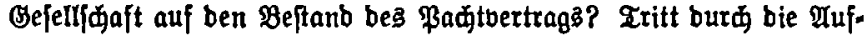
Iöjung bie \&qquibation ein? ..............151

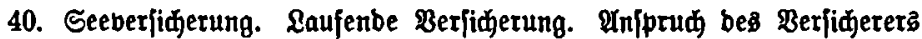

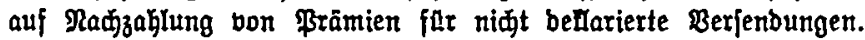

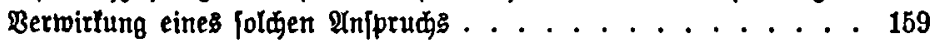

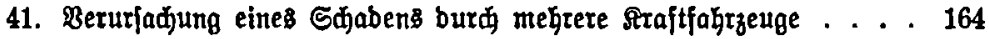

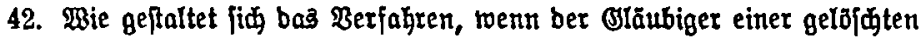

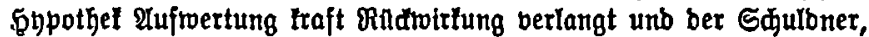

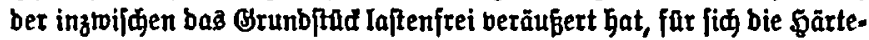
Ilaujel geltenb maden Iann, aber gegen ben Ertwerber bes Grumbituds

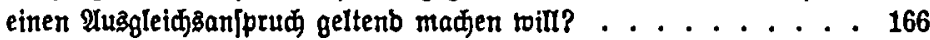

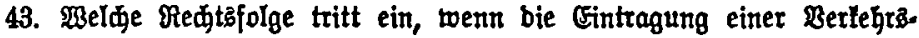
hypothel berwilligt, aber verfehentliđ eine Siderungshypothet cingetrager toorben ift? ............... 
grt.

44. Mietbertrag. Sđ̆riftform. Cinmalige Leiftungen, bie nur alß Bergütung

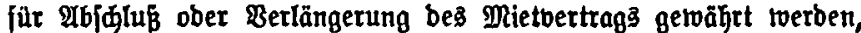
braunen nidt in bie $\mathfrak{u}$ runbe aufgenommen zu twerben . . . . . .

46. $\mathfrak{M u} \beta$ ber Cigentumer einer öffentliden Strafe eine in ben Ruftraum uber ber Strafe Gineinragenbe, polizeilid genegmigte \&iđtrellame.

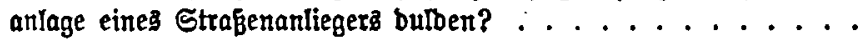

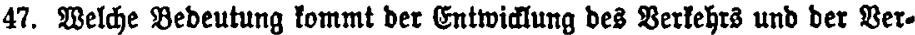
lehramittel für bie Grenzen bes Bemeingebrauds an Straß̧en zu? Staftrogen-Tanftellen ..............

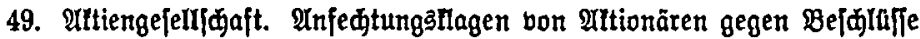
ber Benetalper[ammlung. Unter welden Borausfegungen fant bas

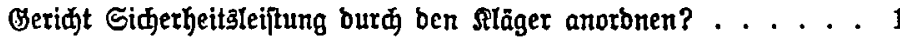

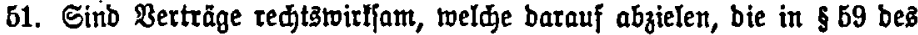

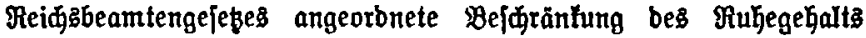
zu verfuten? ...................

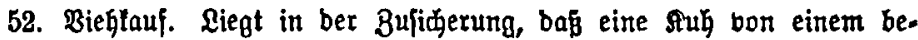
fimmten Tage an eine gewiffe Denge Dild gebe, bie Beteinbarung einer Bewährfrift? Berzug bes Berläuferả mit Burütnahme beß̉

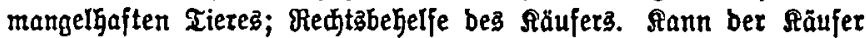

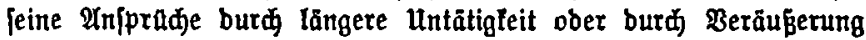
bes Tieres verlieren? ...............

53. Dienftbertrag. Entlafjung eines IIngeftellten. Ulber bie \$aftung

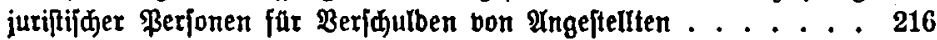

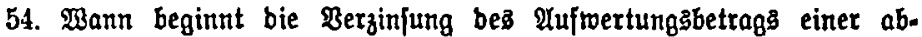

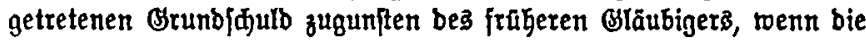

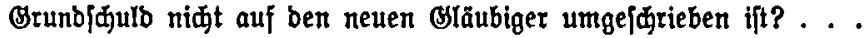

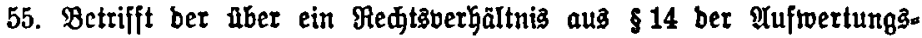
nobelle bom 9. Juli 1927 gefdiofjene Berglei山 aud bann lebiglid

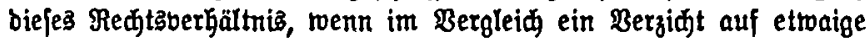

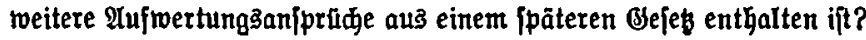

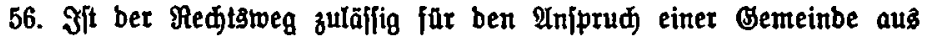
einem ihr gegebenen Beripreden, bie einem Dritten obliegende $\mathfrak{B e r t .}$

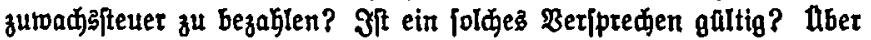

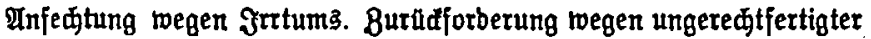

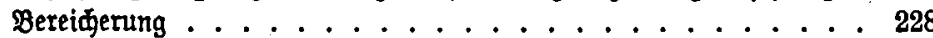


pr.

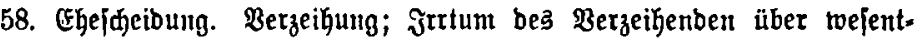

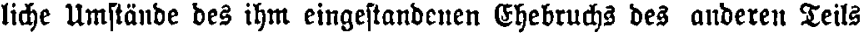

59. Sanu fid cine Bertragşpartei, bie aus einter ifr befamuten Tatjadje feinen Brund zur Riaterfüllung bes Bertraģ entnommen hat, nad)= träglid) zur Ređ)tfertigung igrer Nid)terfüllung auf bieje Tatjade berufen? Wie berbält ẻ fid mit Sd)abenserjab̧anjprüđen, wenn beiben

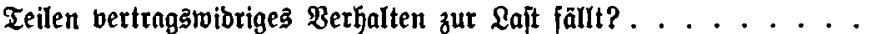

60. Ift aud) ber binglid)e (Hläubiger zur $\mathfrak{A n f e d j t u n g ~ n a d , ~ b e m ~ S i n f e d t u n g ̧ , ~}$

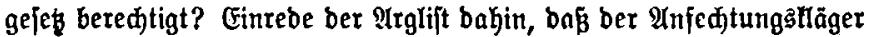
felbjt feituen Sdultitel auf anfectbare \$eife erlangt habe ....

61. (S)enoffenfd)nft mit bejaränfter Şaftpflidgt. Sinb bie Saftfummen ber Benoffen aufzuwerten, wenn bie Benoffenifdaft in Sonfurs gerät unb eine $\mathfrak{u m f t c l l u n g}$ unterbliebcut rar? . . . . . . . . . . . 246

62. Patentred)t. Borbelub̧ungsted)t. über bic Pebeutung ber Begriffe: cine Crfinbung in Benubung nefmen, bie zur Benułung erforber. liden Betanitaltungen trefien ..............

63. Legitimation eites im (égebrud) erzcugten Stindes burd) nadjolgentoc Eje z'wijhen jeiner Mhutter und feinem Erzeuger. Fejtitellung biejer Legitimation burd) bas Bormunbidaftzgerid)t befufs (Fintragung in baż Beburtäregifter . . . . . . . . . . . . . .

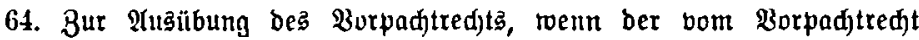
ergriffene Raum zujammen mit anberen Räumen an eillen Dritten verpadtet wirb. Welde Bebeutung haben für bas Borpadtred)t Bebingungen, bie im Bertrng mit ben Dritten filr bie Entftehung bes Redta gelegt find ?

65. Juwieweit obliegt bem Eadjver[tänbigen, ber in einem Etrajuerfahren bernommen worben ift, eine Berpfliøtung zum Sdroeigen über bas,, twas ifm babei zur fienntnis gefommen ift? Sann bie Berlebung

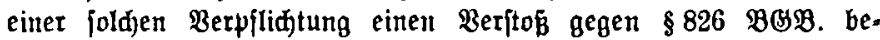
grünben? Jnwierweit ift für ben Tatbeftand bes $\$ 826 \mathfrak{B S B}$. bas Betwuktiein unb bie Millenżridtung bez \$andelnben von Bebeutung?

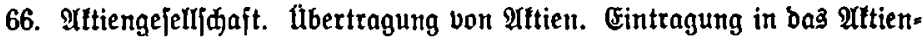
bud . . . . . . . . . . . . . . .

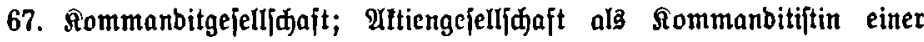

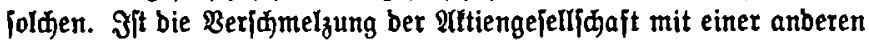


Rv.

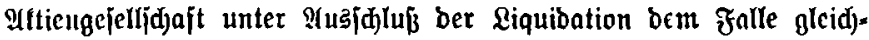
zuftellen, baß eine natürlidje Perjon als Jiommanbitift mit Iob abgeht? Wber bie Bertretung von Bejelljdaftern einer Sommanbitgefellidaft in ber Bejellfáfterberjammlung ............

68. $3 a n n$ Gat bie Finterlegung im Enteignungzberjałren bcfreienbe Sisitfung?

70. Darf ein Unterjaguigsantrng wegen Berlefung bon urheberrechten jo allgemein gefaß̧t fein, baß̄ bie Beftimmung bes umfanges bes Berbots ganz bem 3 wangsbollftredungžberjahren überlaffen bleibt?

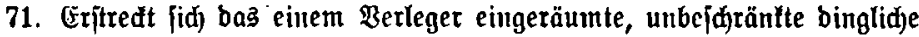
Hrheberred)t aud auf bic erft ipäter aufgefommene Eenbung burd) Miunbunt ober jteht bie giundjunffendung bem urkeber zu? ....

72. llber bie Berpflid)tung bes Eeeverfid)erungsunternefgmers zur forg.

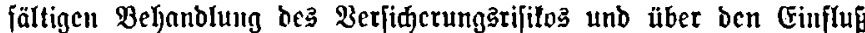

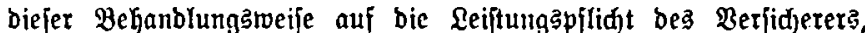
namentlid bci Saftungsbejd̆ränlungen bcr Reeberci. Stann fid̆ bic Piceberei, aud welln ein fog. reines fionnoffement ausgeftellt ift, auj Bermerle im Siai=Empjangs̄jhein berufen?..........

75. Muß (id) eitre \$ypothetenbant nad) 3twangaberfteigerung eines bon ihr bclieberen Grumbjtüđs gefallen Inffen, baß fie im faufgelb= belegungstermin von cinem nadjtebenben Häubiger für ihre nid)t vom (Erfteher za übernehmende நgypothet in Liquibationsgolopjand= briciclt abgefundell miro?

76. Jntwiemeit ift bei einer abgetretenen Sypotheł eit vom ncuen Bläubiger geid)loliener $\mathfrak{A}$ ifwertungäbergleid) gegen ben früheren (Släubiger toirl: jam? Finbet bie Borjdrift in $\$ 67$ Abj. 2 श(1fwes. aud auf Bergleidje Strwelloung, burđ bie ber (stäubiger bie ifm gefeblid) zultehenbe Aujwertung ober mehr als ifren Betrag erhalten hat? . . . . .

77. Saum eine Etabtgemeinbe, bie eine \&ujtbarleit引jteuer erhebt, von ben Theaterunternekmern, benen fie eine aus Feuerwehrleuten beftehende Feuermade ftellt, cine Bergütung nađ burgerlidredtliden Bor¡driften über ben Dienftuertrag verlangen? . . . . . . . .

78. Эit $\mathfrak{A}$ ufrednung mit einer Begenforberung zuläffig, über bie nađ früferer Bereinbarung ber ßarteien ein Edjebsgeridt entjoeiben joll?

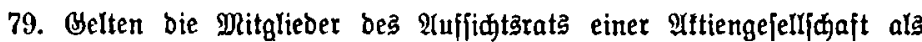
Irbeitnehmer im Sinte bon $\$ 65$ Aufros.? Fallen Ginlagen bes 
nex.

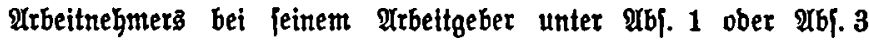
Saß 1 bon $\$ 63$ थuftoß.? . . . . . . . . . . . 351

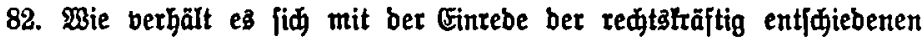
Sahe, wenn in einem Borprozes bie Slage wegen mangelnber Sad. befugniz unb - bei unterftellter Saðbejugnis - auめ als unbegrunbet abgewiefen worber ift? fann bie Cinrebe auj beibe abbeijungş.

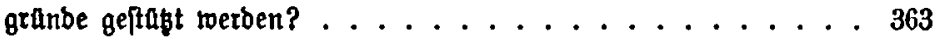

84. Jann nady bem Tobe eines mit Ginterlaffung mehrerer Erben ver. ftorbenen Sommanbitiften an jeiner Stelle bie Grbengemeinjajaft als Pommanbitiftin im Sanbelšregifter eingetragen werben? . . . . .

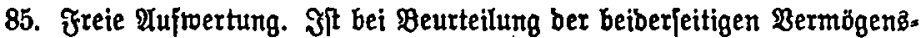

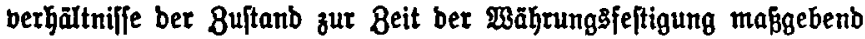

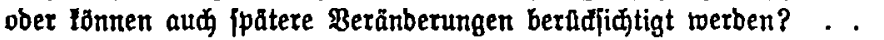

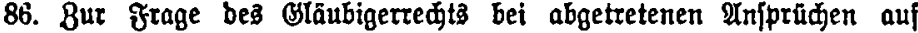

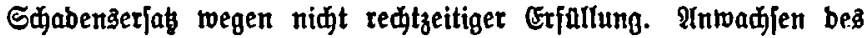

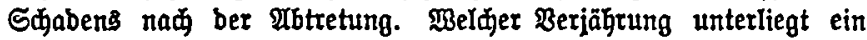

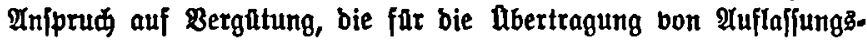
redten an Brumbltuden vereinbart ift? . . . . . . . . . 378

87. Bum Begriff bes Sontolorrents ober einer anberen Iaufenben Bied.

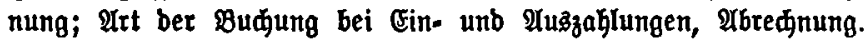
Bum Begriff ber Bermőgensanlage unb bes Beteiligungāberhăltniffesె nă bem $\mathfrak{A} u$ fruertungsgefeg . . . . . . . . . . . 384

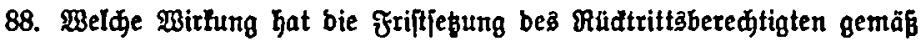

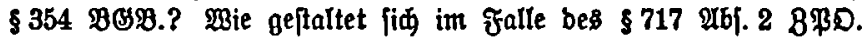

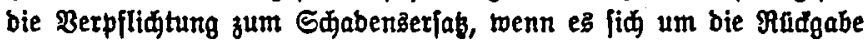
einer im Bege ber Bwangabollftredung weggenommenen Sad. gefamtheit Ganbelt? ............... 388

89. Unter welden umianben tann ein Tilgungşplan als Darleherts.

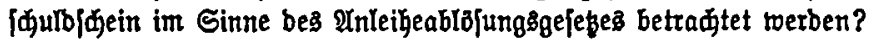

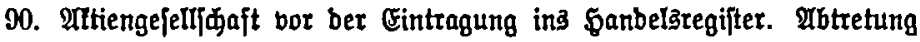

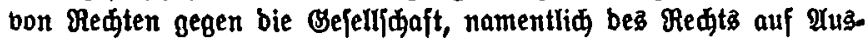
bänbigung von âtien. âbertragung bon Anteiläredten . . . . 401

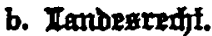

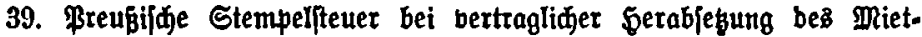
ober Pađtzinfes. Maffen naw ber preubifien Sagborbnung aud 


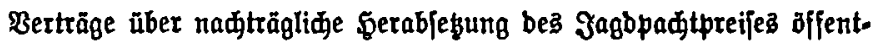
lid ausెgelegt retben?

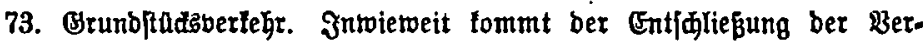
waltungăbeĞrbe aber Crteilung ober Ber|agung ber B̋enehmigung rffatwirlenbe Araft zน?

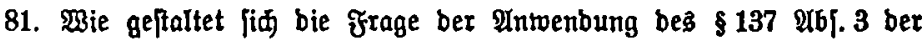
preup. Areisorbnung far bie bftlidjen ßrobinzen bom 13. Dezember

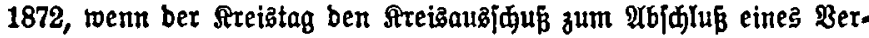
trag̉ fâr ben Areỉ bebollmähtigt? ...........

\section{D̈ffentlides Rent.}

29. Sann nam bem Induftrie-Belaftung gefes eine Berteilung ber $\mathfrak{B e}$ -

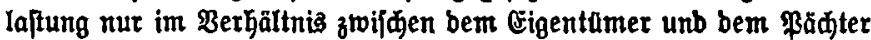
eines Betriebes berlangt werben ober aud im Berhăltnis zroijめen bem leb̆teren unb einem unterpådter ............ 117

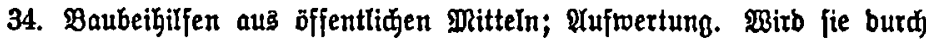
Erhebung von \$auszinsfteuer aubgefdloffen? ......... 138

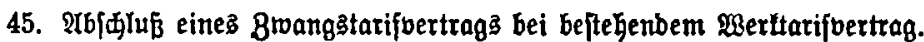

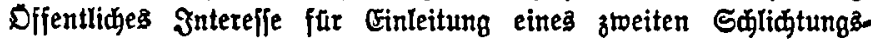

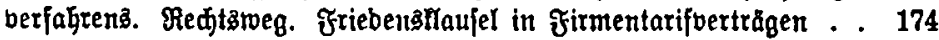

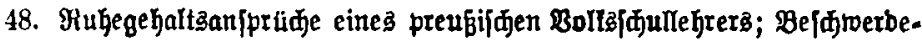
berfahiren. Cinlegung ber Bejdroetbe bet einer unzuftänbigen Stelle

91. Ift bie ఇerorbnung vom 13. Februar 1924 zur Inberung bes

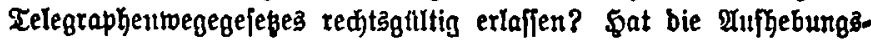
verorbnung bom 18. Oltober 1924 rúdtoirfenbe Staft? . . . . 406

\section{Geribtlide Berfabrex.}

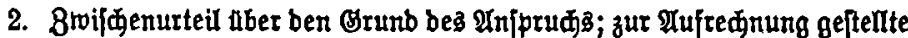
Gegenforberungen; 8urúdbehaltunģ̨redt ..........6

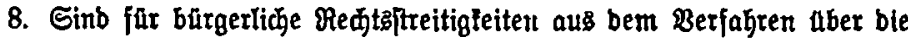
Bitbung von Rentengutern bie orbentliden Beridte ober bie Ranbeg. lulturbeforben zuftånbig? ............... 27

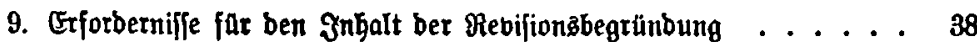


Re.

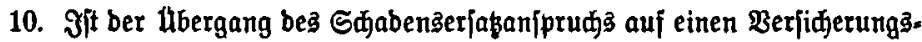

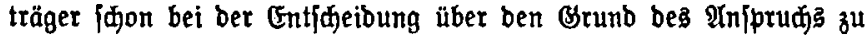
berüdfidtigen? ...............

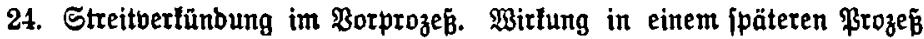

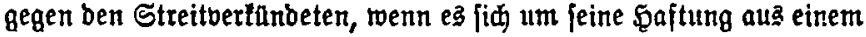
unfall hanbelt, bie $\mathfrak{A}$ rt ber \$aftung aber eine anbere ift als im Bor.

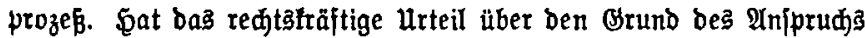
gegenüber bem Streitberfünbeten bie Jnterbentionstwirlungen aud)

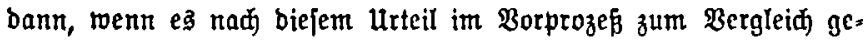
fommen ift? .................

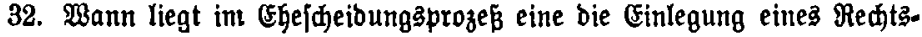
mittels redtfertigenbe Bejatwer bor? ...........

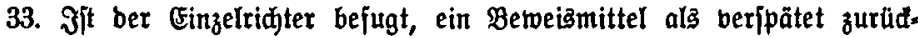

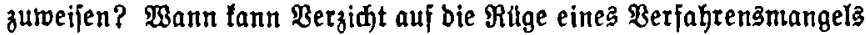
angenommen werben?...............

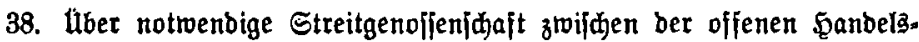
gejelliaft unb einem Befellphafter . . . . . . . . . . . 151

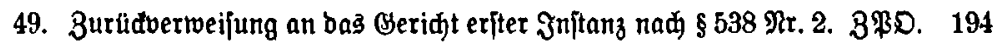

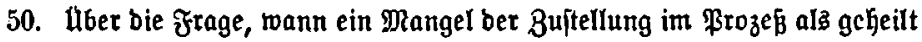
angejeken werben lann. Burüdvertweifung an bas Beridt erfter Initanz nad) $\$ 539$ BPD. . . . . . . . . . . . . . . 204

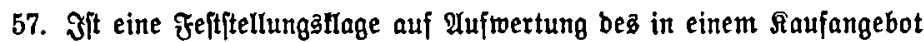
beftimmten Raufpreifes zuläffig, wenn babei nod in ber Butunit liegende Bethältniffe mit in Betradt fommen? .........

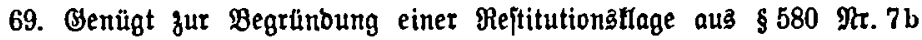
390. gegen ein urteil eriter Snjtanz eine Urtumbe, bie er[t nad ber

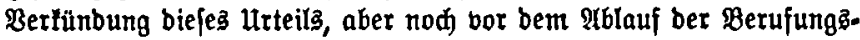
frift erriditet toorben ift? . . . . . . . . . . .

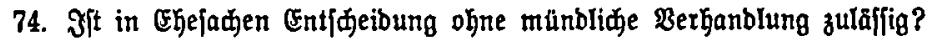
Sann bei einem auf Sheibung lautenben $\mathfrak{u}$ rteil eine einmalige $8 \mathfrak{H}$ ftellung beş ganzen urteils joroohl bie Bettünbigung erjeşen als aud) bie Redtsmittelfrift in Lauf feßen? . . . . . . . . . .

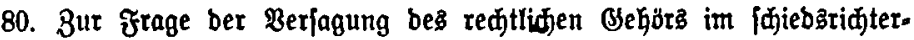
liden Berfahren .................. 
9r.

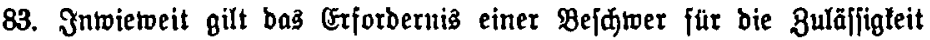

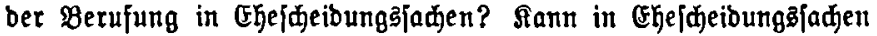
Berufung lebigliđ zum Brwede ber Erthebung einer Miberflage eins gelegt werben? . . . . . . . . . . . . . 364

\section{Anbang: Catideibangen bes Stantsgerintshofs.}

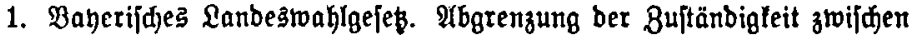

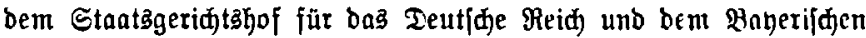

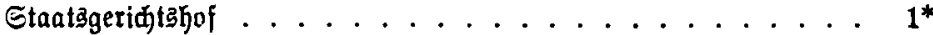

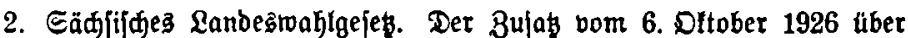

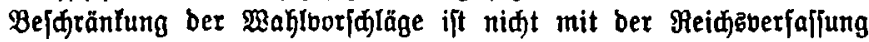
vereinbar. Ungultigleit ber auf biejer Brunblage vorgenommenen żahlen .................... 13*

Eadregiiter ................... 413

Gejęeḡregifter . . . . . . . . . . . . . . . . 424

Bufammenjtellung nadj ber Beitjolge . . . . . . . . . 434

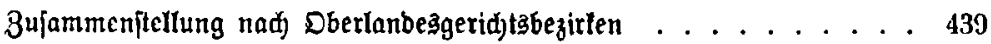

Beridtigungen . . . . . . . . . . . . . 440 
